Gynäk. Rdsch. 1967;4:204-205

\title{
Weitere Referate
}

Ing. St. Holänek, Forschungsanstalt für technische und apparative Ausstattung von

Krankenhäusern (Direktor: Dr. J. Kozumplik), und Dr. J.Srácek, I.Universitäts-Frauenklinik (Direktor: Prof. Dr. M. Uher), Brünn

berichten über verschiedene Kunststqffe, die zur Herstellung von intrauterinen Pessaren geeignet sind.

R. St£rba und Mitarbeiter, Forschungsinstitut für Naturheilmittel, Prag, und Kliniken in Prag, Olmütz, Ostrau und Trencínë

berichten über Erfahrungen mit der zweiphasigen hormonalen Antikonzeption mit Antigest B.

K. Dvorak und Mitarbeiter

geben eine ausführliche zusammenfassende Übersicht über Erfahrungen mit Antigest aus 12

Kliniken bei 877 Frauen mit 5107 לyklen.

F. Havránek, M. Tichý und H. Dyková, Institut für Mutter- und Kinderfür-sorge (Direktor: Prof.

Dr. J. Horský), Prag

haben Antigest A an 20 infertile Frauen mit bisher biphasischen Zyklen verab-reicht sowie

Antigest B an 20 fertile Frauen. Es wurden Endometriumbiopsien zwischen dem 14. und 27.

Zyklustag gemacht. Die histologischen Veränderungen werden mit-geteilt.

Weitere Referatc

205

J. Soska und J. Sráõek, I. Universitäts-Frauenklinik (Direktor: Prof. Dr. M. Uher), Brünn sprachen über organisatorische und ökonomische Fragen der Anwendung der intraute-rinen Antikonzeption.

M. Chalupa, I. Universitäts-Frauenklinik (Direktor: Prof. Dr. M. Uher), Brünn

hat bei arabischen Frauen in Tunesien die Lippes-Schlinge angewendet und gute Er-fahrungen gemacht. Es ist nötig, Größe und Art der Schlinge individuell anzupassen.

J. Antos und O. Cech, Gynäkologische Abteilung des Kreiskrankenhauses (Ghefarzt: MUDr. J. Antos), Sumperk

haben eine übersichtliche Dokumentation über die Antikonzeptionsberatung der Gynäkologen auf Lochkarten A 5 ausgearbeitet, die für praktische und wissenschaftliche Zwecke empfohlen wird.

K.7/8. $7 / 8$ Áк und M. Molcík

II. Universitäts-Frauenklinik (Direktor: Doz. Dr. J. Stanicek) und Institut für Metallforschung der Tschechoslowakíschen Akademie der Wissenschaften

Brünn

zeigen elektronenmikroskopische Studien der Oberfläche des AntikoПzeptionskörper-chens. 